\title{
Quasi-periodic oscillations in solar X-ray sources
}

\author{
Hana Mészárosová ${ }^{1}$, Marian Karlický ${ }^{1}$, and František Fárník ${ }^{1}$ \\ ${ }^{1}$ Astronomical Institute of the Academy of Sciences, \\ CZ-25165 Ondřejov, Czech Republic \\ email: hana@asu.cas.cz
}

\begin{abstract}
We have searched for quasi-periodic oscillations in the hard X-ray emission of solar flares. We have selected 14 flare events which were divided into two groups: a) the events with the X-ray sources located at the flare loop footpoints and b) the events with the X-ray source above the solar limb, i.e. with the loop-top X-ray source. We found that while in the case with the footpoints X-ray sources the quasi-periods of the recorded oscillations were in the interval 2$380 \mathrm{~s}$, in the events with loop-top sources only the quasi-periods longer than $50 \mathrm{~s}$ were recognized. These results are probably connected with the MHD oscillation modes of the flaring loop. While the long periods, which are dominant in loop-top sources, are produced by acoustic oscillations along the whole long loop, in the layers close to the loop footpoints also the MHD wave modes in shorter structures with shorter periods are generated.
\end{abstract}

Keywords. Sun: flares, Sun: X-rays, Sun: oscillations, methods: data analysis

\section{Introduction}

As presented in the papers by e.g. Aschwanden (2004), Mészárosová et al. (2006), Nakariakov \& Melnikov (2009), Karlický et al. (2010), the quasi-periodic oscillations with periods from seconds to tens of minutes are often observed in solar flares, especially in the radio and X-ray bands. It is commonly believed that these oscillations are generated by various types of MHD waves or by periodic magnetic reconnection processes. Among various possibilities for long period oscillations, the most promising mode is the acoustic standing harmonic mode generated along the whole length of the loop. The estimated period is then $P / s=6.7 \times(L / M m) /(T / M K)^{1 / 2}$, where $L$ is the length of the loop and $T$ is the mean loop temperature (Nakariakov et al. 2004). The standing harmonic acoustic mode is connected with the plasma density variations, especially at the loop top, therefore this mode can be directly observable in the flare loop X-ray emissions (because the $\mathrm{X}$-ray emission flux is proportional to the plasma density).

\section{Observations and analysis}

We have analyzed 14 solar hard X-ray flare emissions (Table 1) observed by the Czechmade Hard X-Ray Spectrometer (HXRS, launched onboard the U.S. MTI satellite) with the $0.2 \mathrm{~s}$ time resolution. For a comparison the HXT/Yohkoh (hard X-ray emissions, $0.5 \mathrm{~s}$ time resolution) of the same individual events were used. We have studied quasi-periodic oscillations in the solar X-ray sources located in the footpoints (Table 1, No. 1-7) and in the tops of flaring loops (Table 1, No. 8-14), where locations of the X-ray sources were derived from SXT/Yohkoh (soft X-ray) images. All the emissions quoted in Table 1 were analyzed in detail using the wavelet method (R. Sych, http://pwf.iszf.irk.ru/). The shortest detectable periods depend on the data time resolution (minimal period $P=1 \mathrm{~s}$ 

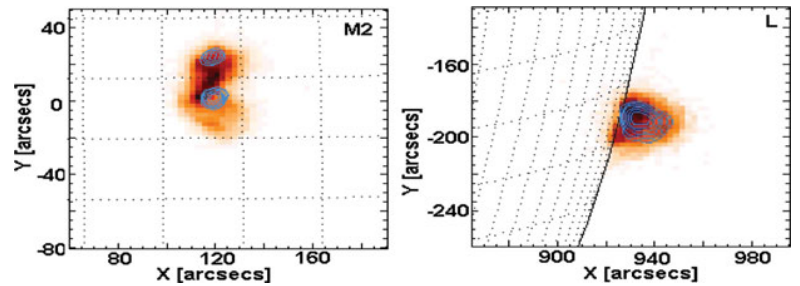

Figure 1. Examples of the SXT/Yohkoh soft X-ray image of the July 25, 2000 event (left panel) and of the July 26, 2000 event (right panel) with superimposed HXT/Yohkoh hard X-ray sources of the M2 and L energy bands located in the footpoints and the top of the flaring loop, respectively.

Table 1. Basic parameters and the characteristic periods $P$ for HXRS/MTI emissions observed in: a) footpoints of flaring loop (No. 1-7, only $P<150$ s are present) and b) top of flaring loop (No. 8-14).

\begin{tabular}{|c|c|c|c|c|}
\hline No. & Event & $\begin{array}{l}\text { Time interval } \\
{[\mathrm{UT}]}\end{array}$ & $\begin{array}{l}\text { Energy band } \\
{[\mathrm{keV}]}\end{array}$ & $\begin{array}{l}\text { Periods } \\
{[\mathrm{s}]}\end{array}$ \\
\hline 1 & Mar 31, 2000 & $10: 15: 05-10: 25: 13$ & $29.0-100.2$ & $5,7,14,32,48,75,87$ \\
\hline 2 & Jul 25, 2000 & $02: 46: 47-02: 54: 03$ & $29.0-100.2$ & $8,11,22,45,66$ \\
\hline 3 & Sep 30,2000 & $23: 16: 50-23: 30: 46$ & $29.0-100.2$ & $11,13,17,27,57,81,115$ \\
\hline 4 & Oct 01,2000 & $13: 59: 24-14: 05: 31$ & $29.0-44.0$ & $14,28,34,52,73$ \\
\hline 5 & Nov 26,2000 & $16: 37: 25-17: 02: 23$ & $44.0-100.2$ & $10,14,26,32,43,50,85,110$ \\
\hline 6 & Jun 05, 2001 & $04: 44: 25-04: 49: 25$ & $12.6-67.2$ & $17,27,38,60,76$ \\
\hline 7 & Aug 31, 2001 & $10: 39: 00-10: 42: 21$ & $29.0-100.2$ & $2,5,12,25,40,50,68$ \\
\hline 8 & Jul 09, 2000 & $07: 19: 4$ & $12.6-29.0$ & 60,170 \\
\hline 9 & Jul 26, 2000 & $07: 41: 42-07: 46: 42$ & $19.0-44.0$ & 54,55 \\
\hline 10 & Sep 30,2000 & $20: 12: 34-20: 17: 34$ & $12.6-29.0$ & $55,68,100$ \\
\hline 11 & Sep 30,2000 & $23: 16: 50-23: 30: 46$ & $12.6-29.0$ & $98,210,280$ \\
\hline 12 & Dec 27,2000 & $15: 35: 39-15: 45: 13$ & $12.6-29.0$ & $90,115,145,190$ \\
\hline 13 & Jun 13, 2001 & $11: 35: 29-11: 48: 15$ & $12.6-29.0$ & $55,65,130,155,190,260$ \\
\hline 14 & Sep 24, 2001 & $10: 07: 02-10: 44: 59$ & $12.6-29.0$ & $130,200,270,300,450$ \\
\hline
\end{tabular}

here) and the longest ones on the length of the individual time series. As likelihood periods are considered periods out of the "cone of influence" (hatched regions in the magnitude wavelet spectra) effected by a finite length of time series. We have found that the hard X-ray emissions located at the footpoints of flaring loop usually show quasiperiodic oscillations with the characteristic periods in whole period range under study $P=2-380$ s (Table 1, No. 1-7). But the emissions located in the top of loops show oscillations only with the period $P$ longer than about $50 \mathrm{~s}(P=54-450 \mathrm{~s}$, Table 1 , No. 8-14). The shorter periods $(P<50 \mathrm{~s})$ are missing in these events.

Here, we present two examples showing both the cases. The left part of Figure 1 shows the SXT/Yohkoh image of the July 25, 2000 event with superimposed HXT/Yohkoh sources (M2 energy band) located in the footpoints of the flaring loop (where M2 = 32.7$52.7 \mathrm{keV}$ ). On the other hand, the left part of Figure 2 presents the corresponding hard X-ray time series (HXRS/MTI, upper panel) and magnitude wavelet spectra with the periods in the broad range, i.e. also with the periods below $50 \mathrm{~s}$. Furthermore, the right part of Figure 1 shows the SXT/Yohkoh image of the July 26, 2000 event with superimposed HXT/Yohkoh source (L energy band) located in the top of the flaring loop (where $\mathrm{L}=13.9-22.7 \mathrm{keV}$ ). The corresponding time series (HXRS/MTI, upper panel) and wavelet spectra are shown in Figure 2 (right part, middle and bottom panels). These wavelet spectra show only the periods greater than $50 \mathrm{~s}$. Any shorter periods are missing. 

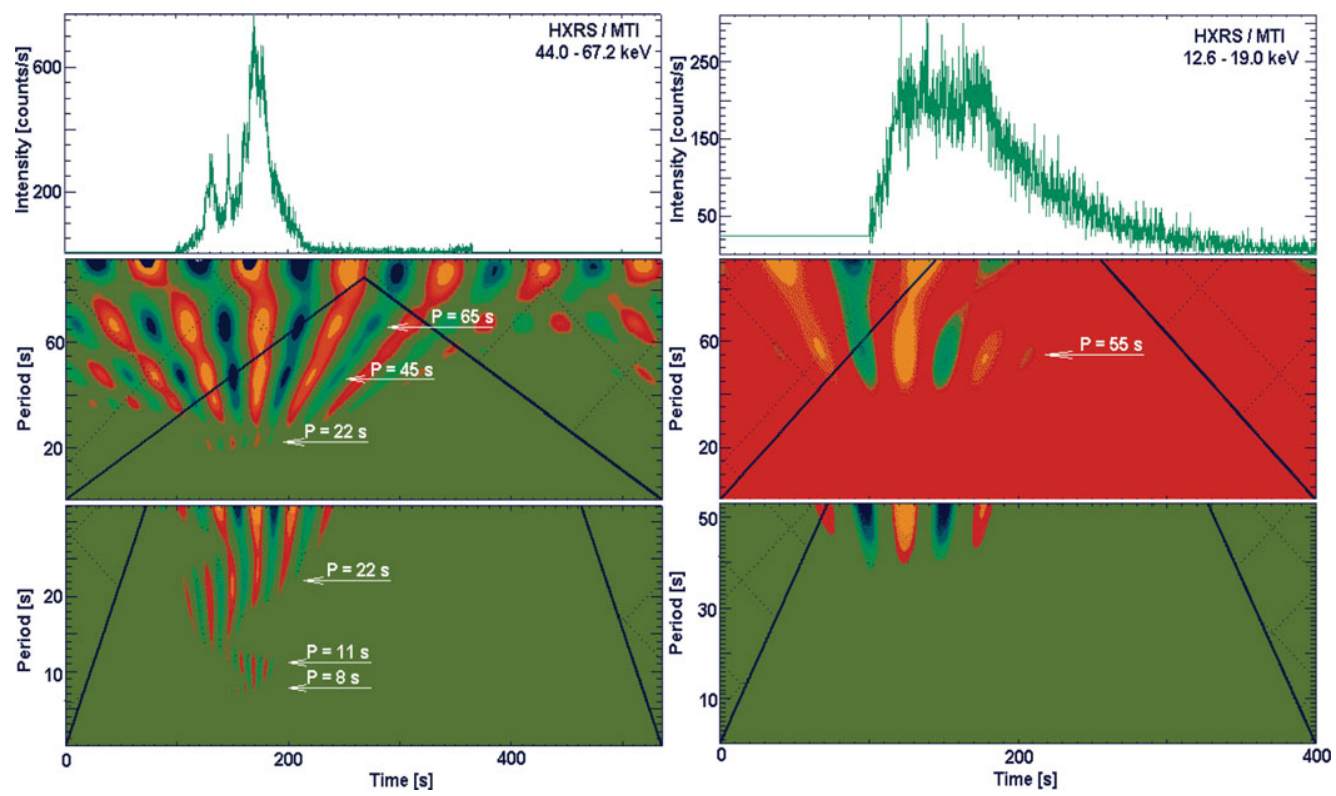

Figure 2. Example of the hard X-rays time series (HXRS/MTI, upper panels) and magnitude wavelet spectra (middle and bottom panels) with the characteristic periods for emissions: a) July 25, 2000 of the flaring loop footpoints (left panels). The energy band is 44.0-67.2 keV. ii) July 26, 2000 of the top of flaring loop (right panels). The energy band is $12.6-19.0 \mathrm{keV}$.

\section{Conclusions}

We have found that the oscillations in solar hard X-ray emissions from the flare loopfootpoints and from the loop-tops differ in the range of periods. The footpoint emissions revealed the quasi-periodic oscillations with characteristic periods $P<50$ s in the wavelet spectra. Such periods were missing in the emissions from the loop-top X-ray sources. The results obtained for the HXRS/MTI emissions were confirmed by those obtained by the analysis of the HXT/Yohkoh data at similar energy bands. These results are probably connected with the MHD oscillation modes of the flaring loop. While the long periods, which are dominant in loop-top sources, are produced by acoustic oscillations along the whole long loop, in the layers close to the loop footpoints also the MHD waves modes in shorter structures with shorter periods are generated.

\section{Acknowledgements}

H. M. and M. K. acknowledge support from the Grant IAA300030701 of the Academy of Sciences of the Czech Republic and the research project AVOZ10030501 of the Astronomical Institute AS CR. The authors also thank Dr. R. Sych for the wavelet package used in this study.

\section{References}

Aschwanden, M. J. 2004, Physics of the Solar Corona, Berlin-Springer Praxis Books Karlický, M., Zlobec, P., \& Mészárosová, H. 2010, Solar Phys., 261, 281

Mészárosová, H., Karlický, M., Rybák, J., Fárník, F., \& Jiřička, K. 2006, A\&\&A, 460, 865

Nakariakov, V. M. \& Melnikov, V. F. 2009, Space Sci. Revs, 149, 119

Nakariakov, V. M., Tsiklauri, D., Kelly, A., Arber, T. D., \& Aschwanden, M. J. 2004, A\&A, $414, \mathrm{~L} 25$ 